Quim. Nova, Vol. 36, No. 2, 306-313, 2013

\title{
FÁRMACOS MULTIFUNCIONAIS: MONOAMINA OXIDASE E $\alpha$-SINUCLEÍNA COMO ALVOS TERAPÊUTICOS NA DOENÇA DE PARKINSON
}

\author{
Cristian Follmer* \\ Departamento de Físico-Química, Instituto de Química, Centro de Ciências Matemáticas e da Natureza, Universidade Federal do \\ Rio de Janeiro, 21941-909 Rio de Janeiro - RJ, Brasil \\ Heleno J. C. Bezerra Netto \\ Instituto Nacional de Propriedade Industrial, Praça Mauá, 7, 20081-240 Rio de Janeiro - RJ, Brasil
}

Recebido em 28/4/12; aceito em 7/8/12; publicado na web em 6/2/13

\begin{abstract}
MULTIFUNCTIONAL DRUGS: MONOAMINE OXIDASE AND $\alpha-S Y N U C L E I N$ AS TARGETS FOR THE TREATMENT OF PARKINSON'S DISEASE. Parkinson's disease (PD) is a neurodegenerative disorder associated to selective degeneration of dopaminergic neurons caused by an intricate relationship among dopamine metabolism, oxidative stress and $\alpha$-synuclein fibrillation. Most therapies for PD have focused on dopamine replacement through the use of both monoamine oxidase inhibitors (MAOIs) and dopamine precursor L-dopa. Interestingly, certain MAOIs have a broad spectrum of action including anti-fibrillogenic properties in $\alpha$-synuclein aggregation. Herein we revisit the chemical properties of MAOIs and their action on important targets associated with PD, notably $\alpha$-synuclein fibrillation and dopamine metabolism, discussing the strategies associated with the development of multitarget drugs for neurodegenerative diseases.
\end{abstract}

Keywords: monoamine oxidase; $\alpha$-synuclein; Parkinson’s disease.

\section{INTRODUÇÃO}

A doença de Parkinson (DP) afeta de 1-2\% da população adulta acima de 65 anos de idade, sendo caracterizada pela degeneração seletiva de neurônios dopaminérgicos na região da substância nigra pars compacta e pela deficiência de dopamina no striatum, sendo a desordem neurológica, associada ao envelhecimento, mais comum após a doença de Alzheimer. ${ }^{1}$

O tratamento farmacológico para DP consiste basicamente no controle sintomático via restituição de dopamina (DA) [4-(2-aminoetil)benzeno-1,2-diol], principalmente através da administração de seu precursor L-DOPA [ácido (S)-2-amino-3-(3,4-di-hidroxifenil propanoico)] ou através do uso de inibidores da enzima monoamina oxidase (IMAO) ${ }^{2,3}$

Muitos dos IMAO utilizados na clínica são irreversíveis, ligando-se covalentemente à enzima e inativando-a, o que resulta em importantes efeitos colaterais, principalmente devido a interações com fármacos simpatomiméticos e com alimentos contendo tiramina, o que resulta em reações hipertensivas severas. ${ }^{4}$ Além disso, IMAO irreversíveis podem apresentar uma série de outros efeitos colaterais indesejáveis no sistema nervoso central (insônia, irritabilidade, agitação, hipomania, supressão do sono REM), bem como disfunções cardiovasculares (hipotensão ortostática) ou distúrbios sexuais. ${ }^{5}$ Assim, o desenvolvimento de novos IMAO tem como desafio a busca por inibidores potentes e com efeitos colaterais reduzidos ou inexistentes.

Outro mecanismo importante associado à DP é o mau enovelamento proteico, levando à formação de inclusões fibrilares intracelulares, constituídas principalmente pela proteína $\alpha$-sinucleína. A $\alpha$-sinucleína é uma proteína de função ainda não totalmente elucidada, que apresenta diversos estados de agregação in vitro, incluindo desde oligômeros pré-fibrilares (protofibras) - capazes de formar poros em membranas fosfolipídicas - até estruturas fibrilares estáveis do tipo amiloide. ${ }^{6}$ Mutações na proteína, principalmente Ala30Pro e Ala53Thr, estão ligadas a casos de DP em grupos de indivíduos

*e-mail: follmer@iq.ufrj.br mais jovens. ${ }^{7,8}$ Além da DP, a formação de agregados proteicos é observada numa série de doenças neurodegenerativas como a doença de Alzheimer (agregação do peptídeo $\beta$-amiloide e da proteína tau), encefalopatias espongiformes (agregados da proteína do príon) e doença de Huntignton (agregação da huntingtina). ${ }^{9}$

Estudos recentes mostram que alguns IMAO, como a selegilina, apresentam outros alvos moleculares importantes além da inibição enzimática, como atividades antioxidante e antifibrilogênica - esta última associada à inibição da formação de fibras de $\alpha$-sinucleína. Nesta revisão, as bases moleculares do desenvolvimento de fármacos multialvos para a DP são exploradas, visando entender os aspectos químicos da atividade inibitória sobre MAO combinada com propriedades antioxidantes e antifibrilogênicas exibidas por estes compostos.

\section{A doença de Parkinson, dopamina e estresse oxidativo}

Um dos fatores associados à neuropatogênese da DP é o estresse oxidativo decorrente da excessiva geração de espécies reativas do oxigênio (ROS). ${ }^{10}$ Apesar de concentrações baixas de ROS estimularem vários processos celulares e regularem algumas importantes funções fisiológicas, estas espécies podem causar um aumento na peroxidação lipídica e modificações químicas em proteínas e ácidos nucleicos e, com isso, resultar em neurodegeneração. ${ }^{11}$ Uma vez formadas, as ROS podem abstrair hidrogênio do DNA formando DNA radicalar, que leva a uma fragmentação do DNA, depleção de NADH e ATP e, por fim, apoptose. ${ }^{12}$ Neste contexto, o estresse oxidativo associado ao metabolismo de DA tem sido sugerido como um dos principais mecanismos envolvidos na produção de ROS e metabólitos tóxicos nos neurônios dopaminérgicos. Assim, DA funcionaria como uma neurotoxina endógena, aumentando a exposição dos neurônios dopaminérgicos a danos oxidativos - um dos principais fatores fisiopatológicos envolvidos na DP. ${ }^{13}$

Na oxidação não enzimática, DA sofre a perda de um elétron formando radical dopamina semiquinona capaz de oxidar proteínas e modificar DNA. A subsequente perda de mais um elétron leva à formação de dopamina orto-quinona, capaz de reagir com 
importantes nucleófilos celulares como tióis e proteínas. Esta reação é fortemente favorecida por $\mathrm{pH}$ alcalino; a DA orto-quinona sofre ciclização via reação de Michael, a qual é favorecida por $\mathrm{pH}$ ácido, levando à formação de leucodopaminocromo $(1 \mathrm{H}$-indol-5,6-diol $) .{ }^{14} \mathrm{O}$ resultado destas reações é a formação espontânea de semiquinonas e quinonas potencialmente tóxicas para as células, as quais são capazes de polimerizar gerando neuromelamina, além de ROS (Figura 1A). ${ }^{15}$ Além disso, a presença de metais e/ou oxigênio molecular favorece a formação de ROS. Já na oxidação enzimática da DA por ação das enzimas MAO-A ou MAO-B, além da formação do aldeído correspondente, DOPAL (3,4-di-hidroxifenilacetaldeído) (Figura 1B), há a geração de peróxido de hidrogênio que, em presença de $\mathrm{Fe}^{+2}$, pode gerar radicais hidroxilas altamente reativos e citotóxicos via reação de Fenton (Reação 1):

$\mathrm{Fe}^{2+}+\mathrm{H}_{2} \mathrm{O}_{2} \rightarrow \mathrm{Fe}^{3+}+\mathrm{OH}^{-}+{ }^{\cdot} \mathrm{OH}$

(Reação 1)

DOPAL é um aldeído altamente reativo, capaz de reagir com proteínas via formação de base de Schiff, apresentando toxicidade in vivo e in vitro de $100-1000$ vezes maior que a observada para DA. ${ }^{16}$ A formação de ROS na oxidação do DOPAL pode explicar em parte sua grande toxicidade e seu envolvimento na DP. A Figura 1B mostra que superóxido é produzido durante a oxidação do DOPAL via redução sequencial de oxigênio molecular pelo ganho de um elétron.

\section{MONOAMINA OXIDASE COMO ALVO TERAPÊUTICO}

Monoamina oxidase (MAO) [amina: oxigênio oxirredutase; (EC 1.4.3.4)] é uma enzima contendo o cofator flavina-adenosina-dinucleotídeo (FAD) localizado na membrana mitocondrial externa, que catalisa a desaminação oxidativa de monoaminas endógenas ou exógenas (Reação 2):

$\mathrm{RCH}_{2} \mathrm{NH}_{2}+\mathrm{H}_{2} \mathrm{O}+\mathrm{O}_{2} \rightarrow \mathrm{RCHO}+\mathrm{NH}_{3}+\mathrm{H}_{2} \mathrm{O}_{2}$

(Reação 2)

Entre os substratos da MAO encontram-se neurotransmissores (dopamina, serotonina, norepinefrina, tiramina e triptamina) e aminas exógenas, como a neurotoxina 1-metil-4-fenil-1,2,3,6-tetra-hidropiridina (MPTP). ${ }^{17-19}$ Duas isoformas de MAO foram identificadas, MAO-A e MAO-B, as quais apresentam uma identidade de sequência de $72 \%$ e se distinguem quanto à especificidade ao substrato e à inibição. ${ }^{20}$

Uma atividade anormal da isoforma MAO-B está relacionada a várias desordens neurológicas, como DP e doença de Alzheimer, enquanto que a isoforma MAO-A parece estar associada com condições psiquiátricas como depressão e ansiedade. ${ }^{21}$ Assim, diversos IMAO têm sido desenvolvidos visando tanto o tratamento de distúrbios de depressão (inibição da MAO-A), como as terapias sintomáticas da DP e da doença de Alzheimer. ${ }^{21-24}$ Uma redução dos níveis de atividade de MAO resultaria então num aumento nas concentrações de certos neurotransmissores no cérebro, cujo declínio está intimamente ligado ao processo de envelhecimento, além de uma diminuição na produção de peróxido de hidrogênio, subproduto da reação catalisada por MAO. A Tabela 1 apresenta alguns IMAO utilizados na clínica para tratamento de depressão ou DP, cujas estruturas são apresentadas na Figura 2.

Os IMAO apresentam uma série de atividades além do aumento nos níveis de certos neurotransmissores, como atividade antioxidante e antiapoptótica. ${ }^{25,26}$ Visto que MAO-B está envolvida na conversão do MPTP em sua forma tóxica $\mathrm{MPP}^{+}$(1-metil-4-fenilpiridina), entre os efeitos esperados para os IMAO estariam a inibição da conversão de toxinas do tipo MPTP em seus metabólitos tóxicos, acompanhada de
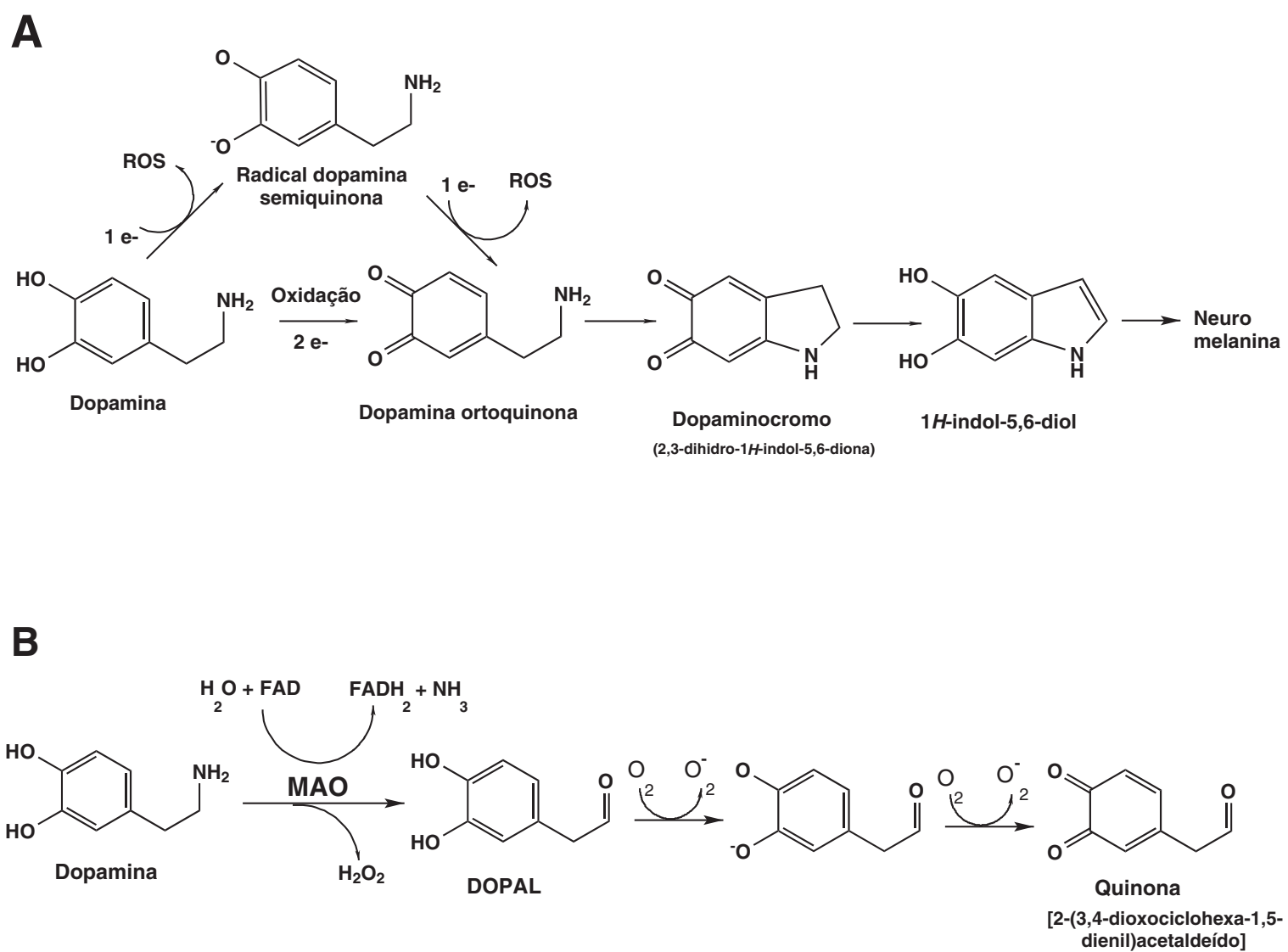

Figura 1. Vias de oxidação da dopamina. (A) Dopamina é oxidada por uma via não enzimática, levando à formação de quinonas e semiquinonas, potencialmente tóxicas para as células, além de ROS; (B) dopamina é metabolizada pela enzima MAO, gerando o aldeído DOPAL, o qual pode sofrer oxidação com liberação de superóxido 
Tabela 1. Propriedades de alguns inibidores de MAO utilizados na clínica

\begin{tabular}{llll}
\hline Inibidor & Seletividade & Modo de ação & Características \\
\hline Clorgilina & MAO-A & Irreversível & $\begin{array}{l}\text { Atividade antidepressiva; aumento dos níveis de noradrenalina e serotonina; efeito colateral: } \\
\text { hipertensão. }\end{array}$ \\
Moclobemida & MAO-A & Reversível & $\begin{array}{l}\text { Atividade antidepressiva; não causa hipertensão; efeitos antissintomáticos suaves em DP. } \\
\text { Selegilina }\end{array}$ \\
MAO-B & Irreversível & $\begin{array}{l}\text { Utilizada na terapia de PD; inibição de MAO-A e atividade antidepressiva em altas doses; não } \\
\text { causa hipertensão; neuroproteção frente ao MPTP; efeitos simpatomiméticos relacionados a } \\
\text { metabólitos de metanfetamina. }\end{array}$ \\
Rasagilina & MAO-B & Irreversível & $\begin{array}{l}\text { Utilizada na terapia de DP; IMAO-B mais potente que selegilina; não apresenta efeitos } \\
\text { simpatomiméticos. }\end{array}$ \\
\hline
\end{tabular}
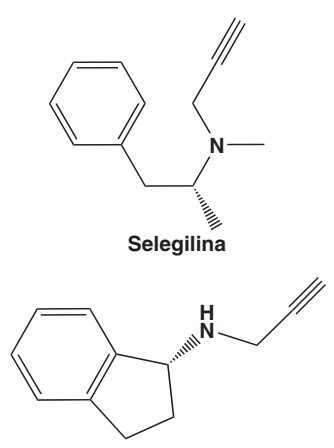

Rasagilina<smiles>C#CCN(C)Cc1ccccc1</smiles><smiles>C#CCN(C)CCCOc1ccc(Cl)cc1Cl</smiles><smiles>COCC(C)(C)OC</smiles><smiles>C#CCN[C@H]1CCc2cc(C)c(OC(=O)N(C)CC)cc21</smiles><smiles>Cc1nccc2c1[nH]c1cc(O)ccc12</smiles><smiles>CC1=C(C)C(=O)c2cc(C)ccc2C1=O</smiles>

Figura 2. Estruturas de alguns inibidores de MAO. Selegilina (inibidor irreversível de $M A O-B$ ); pargilina (inibidor irreversível de $M A O-B$ ); rasagilina (inibidor irreversível de $M A O-B$ ); clorgilina (inibidor irreversível de $M A O-A$ ); moclobemida (inibidor reversível de $M A O-A$ ); ladostigil (inibidor irreversível de MAO-B); isatina (inibidor reversível de MAO-B); harmina (inibidor reversível de MAO-A); TMN (inibidor reversível e não seletivo de MAO)

uma redução na geração de ROS a partir de peróxido de hidrogênio.

No tratamento sintomático para DP, os IMAO são empregados visando o aumento da DA neuronal. DA é metabolizada pela isoforma MAO-A e pela MAO-A/MAO-B em células gliais e astrócitos. ${ }^{26}$ Portanto, a inibição seletiva de MAO-A ou MAO-B não necessariamente resulta em um aumento nos níveis de DA na região do striatum afetada pela degeneração na DP, visto que a diminuição da atividade de uma isoforma específica pode ser compensada por um aumento da atividade da outra isoforma. Por exemplo, estudos em ratos mostraram que a administração dos inibidores irreversíveis selegilina (MAO-B) ou clorgilina (MAO-A) não produziu um aumento de DA na mesma proporção do que o observado para os neurotransmissores feniletilamina, noradrenalina ou serotonina. ${ }^{27}$ Assim, o aumento de DA observado quando um inibidor seletivo de MAO é administrado provavelmente esteja associado a uma modulação dos receptores de DA. ${ }^{21}$ Por outro lado, a administração de IMAO não seletivos como ladostigil ou isocarboxadil resultou em um aumento bastante pronunciado nos níveis de DA. Além disso, inibidores não seletivos podem aumentar os níveis tanto de DA como de serotonina - um resultado importante visto que muitos pacientes com DP apresentam um quadro de depressão associado à doença. ${ }^{21}$

A inibição irreversível de MAO-A está associada à potencialização da atividade cardiovascular simpática, devido a um aumento na liberação de noradrenalina. ${ }^{4}$ Este efeito colateral ocorre quando tiramina, que é efetivamente metabolizada pela MAO-A intestinal, entra na circulação sanguínea, o que resulta na liberação de noradrenalina nos terminais nervosos simpáticos e de adrenalina na glândula adrenal. Este efeito colateral de inibidores irreversíveis de MAO-A é conhecido como "reação a queijo", visto que tiramina e outras aminas simpatomiméticas estão presentes em alimentos e bebidas fermentadas como queijo e cerveja. Portanto, crises hipertensivas podem ser evitadas utilizando-se IMAO-A reversíveis ao invés de irreversíveis. Devido à baixa quantidade de MAO-B presente no intestino, IMAO-B, especialmente aqueles seletivos, não promovem a "reação a queijo" - a não ser quando administrados em doses altas o suficiente para inibir MAO-A.

Os efeitos colaterais associados à inibição irreversível da MAO-A intestinal podem ser evitados utilizando-se inibidores tecido-específicos como o ladostigil (Figura 2), um inibidor seletivo que atua especificamente sobre a MAO-A presente no cérebro. No entanto, as bases moleculares da inibição de MAO tecido-específica ainda não são bem entendidas. Neste contexto, diversas pesquisas têm buscado o desenvolvimento de novos IMAO reversíveis e não seletivos.

\section{ASPECTOS ESTRUTURAIS DA SELETIVIDADE DAS ISOFORMAS DE MAO}

Vários estudos têm contribuído para uma melhor compreensão dos fatores responsáveis pela atividade inibitória sobre MAO e a seletividade MAO-A/B. ${ }^{28}$ As estruturas das isoformas MAO-A e MAO-B humanas, determinadas pela técnica de cristalografia de raios-X, são apresentadas na Figura $1 \mathrm{~S}$, material suplementar. ${ }^{28,29}$ Apenas as cadeias A das enzimas foram representadas para melhor visualização. Pode-se observar que as duas isoformas apresentam estruturas terciárias bastante semelhantes. As regiões correspondentes aos resíduos da porção C-terminal da proteína, 463-506 em MAO-A e 457-501 em MAO-B (destacadas em verde), estão envolvidas no ancoramento da enzima na membrana externa da mitocôndria. Apesar desta região não alterar a especificidade ao inibidor, é fundamental para a atividade catalítica da enzima. Pode-se também observar que o cofator FAD e as duas tirosinas que constituem a "gaiola aromática", Tyr407/Tyr444 em MAO-A e Tyr398/Tyr435 em MAO-B, apresentam estruturas idênticas (Figura 1S, material suplementar). ${ }^{30}$

Apesar desta convergência estrutural das isoformas de MAO, existem particularidades em seus sítios ativos que são responsáveis pela diferença na suscetibilidade à inibição. As maiores diferenças existem na área do sítio ativo na região oposta ao grupamento flavina, envolvida no reconhecimento do substrato. Analisando-se a cavidade 
na enzima envolvida na ligação ao substrato/inibidor, observa-se que dos 16 resíduos presentes nesta região, 6 são diferentes entre MAO-A e MAO-B. A seletividade de inibidores reversíveis está intimamente relacionada com as diferenças no tamanho e forma desta cavidade. MAO-A humana apresenta uma única cavidade para o substrato com $550 \AA^{3}$, a qual é menor em comprimento e largura do que a cavidade em MAO-B com $700 \AA^{3}$, mais longa e estreita. ${ }^{31}$ Outro fator importante é a conformação da alça da região 210-216 da cadeia polipeptídica da MAO-A e 201-206 em MAO-B (Figura 1S, em amarelo no material suplementar). Esta alça, posicionada na entrada da cavidade do sítio ativo, apresenta uma conformação mais estendida e com maior amplitude de movimento na isoforma MAO-A do que em MAO-B. Assim, o sítio de ligação ao inibidor em MAO-A é formado por uma única cavidade que se estende do anel da flavina até a alça 210-216 e, assim como na MAO-B, esta cavidade apresenta um caráter hidrofóbico. ${ }^{28}$ Portanto, a forma e o tamanho das cavidades dos sítios ativos são diferentes entre as isoformas, em decorrência tanto das substituições dos aminoácidos como de alterações conformacionais.

Certos resíduos presentes no sítio de interação da enzima com inibidor/substrato, como Phe208 em MAO-A ou Tyr326 em MAO-B, têm um grande impacto sobre a seletividade da enzima. ${ }^{28}$ Por exemplo, comparando-se os inibidores competitivos e reversíveis harmina (MAO-A) e isatina (MAO-B), cujas estruturas são mostradas na Figura $2 \mathrm{~S}$ do material suplementar, pode-se verificar que a posição dos anéis aromáticos destes inibidores é altamente conservada. As estruturas coplanares dos anéis aromáticos dos inibidores harmina e isatina estão envolvidas em interações do tipo $\pi-\pi$ com o grupo amida da Gln215 na MAO-A e Gln206 na MAO-B, respectivamente. No lado oposto da Gln215/206, os anéis aromáticos destes inibidores interagem com Phe352 e Tyr407 em MAO-A e Phe343 e Tyr398 em MAO-B. Neste caso, a presença da Tyr326 no sítio da MAO-B desfavorece a interação com a harmina por impedimento estéreo. ${ }^{28}$ Estas características estruturais também explicam a diferença de susceptibilidade destas enzimas a certos inibidores irreversíveis, como a clorgilina (MAO-A) ou selegilina (MAO-B), da classe das propargilaminas (Figura 2S). Neste caso, a ligação da selegilina é desfavorecida pelo posicionamento da Phe208 no sítio ativo da MAO-A, enquanto que a ligação da clorgilina no sítio ativo da MAO-B é desfavorecida pela posição da Tyr326. ${ }^{31}$

Alguns outros fatores estruturais são igualmente importantes no desenvolvimento de IMAO e devem ser considerados no desenho destas moléculas: i) lipofilicidade do inibidor, a qual é fundamental para atravessar a barreira hematoencefálica e, assim, inibir a enzima no cérebro; ii) a presença de ligações de hidrogênio entre grupos polares do inibidor e a enzima, principalmente envolvendo o grupamento flavina do cofator FAD ou os resíduos Tyr, Gln, Asn ou Glu presentes no sítio catalítico, estabilizando o complexo inibidor-enzima; iii) a presença de uma estrutura planar do inibidor, que possa melhor se adaptar à fenda catalítica. ${ }^{32}$

Diversos estudos têm buscado isolar e caracterizar IMAO presentes em plantas. Um destes compostos é a 2,3,6-trimetil-1,4-naftoquinona (TMN, Figura 2), um inibidor reversível e competitivo de MAO isolado de folhas de tabaco. ${ }^{33}$ Dados de modelagem molecular sugerem que a TMN interage com a porção flavina do FAD da enzima através de uma ligação de hidrogênio, ${ }^{34}$ como mostrado na Figura 3S, material suplementar. Além disso, os anéis aromáticos da cadeia lateral da Tyr407 e Tyr444 da MAO-A e Tyr398 e Tyr435 da MAO-B desempenham uma importante função na estabilização da estrutura do anel 1,4-naftoquinona, dentro do sítio catalítico, através de interações do tipo London. Estes resíduos de tirosina são conservados tanto em MAO-A como MAO-B de diferentes espécies. ${ }^{35}$ Nesta conformação, o ligante encontra-se localizado entre os dois resíduos de tirosina da enzima, perpendicularmente à face re da porção flavina, permitindo que as cadeias laterais fenólicas formem um "sanduíche aromático". Dados de cristalografia sugerem que tanto os inibidores como os substratos de MAO devem adentrar pela região formada por estas duas tirosinas para interagir com o grupo flavina. ${ }^{36}$ No caso da rasagilina, um inibidor irreversível de MAO-B, a porção aromática do inibidor interage com as cadeias laterais dos resíduos da Tyr398 e Tyr435 na estrutura da MAO-B, enquanto que os resíduos da Tyr60 e Phe343 estariam envolvidos na estabilização do complexo, similarmente ao observado com TMN e MAO-B. ${ }^{37}$

Os estudos com TMN têm sugerido que o grupo 1,4-naftoquinona pode representar um importante farmacóforo para o desenvolvimento de IMAO não seletivos ou pouco seletivos e, principalmente, reversíveis. ${ }^{34} \mathrm{O}$ grupo 1,4-naftoquinona é encontrado em diversas moléculas bioativas, como alguns compostos antitumorais e em vitaminas K. ${ }^{38}$ Menadiona (2-metil-1,4-naftoquinona), uma forma sintética de vitamina $\mathrm{K}$ (vitamina K3), comporta-se como um inibidor reversível de MAO-B, com uma seletividade de 60 vezes para MAO-B humana em comparação com MAO-A ( $\mathrm{Ki}^{\mathrm{MAO}-\mathrm{A}} / \mathrm{Ki}^{\mathrm{MAO}-\mathrm{B}}$ $=60) \cdot{ }^{34} \mathrm{~A}$ seletividade observada para menadiona está na mesma ordem daquela observada para rasagilina, um inibidor irreversível de MAO-B cuja seletividade é de 50-vezes e 93-vezes para MAO-B humana e de rato, respectivamente. No entanto, a seletividade observada para menadiona é significantemente menor que a da selegilina (seletividade de 250 vezes para MAO-B humana ou de rato) - um inibidor irreversível utilizando na clínica para o tratamento de DP. ${ }^{39}$

A menadiona interage com a porção flavina do FAD da MAO-B através de ligações de hidrogênio (Figura 3S, material suplementar) e ocupa uma posição similar à observada para a isatina (indol-2,3-diona), um inibidor endógeno de MAO-B. ${ }^{34} \mathrm{Na}$ isatina, o anel indol encontra-se entre os resíduos Tyr435 e Tyr398 em uma "gaiola hidrofóbica" e em uma conformação perpendicular ao anel flavina do cofator FAD ${ }^{37}$ similar ao observado para menadiona e MAO-B (Figura 3S, material suplementar).

\section{Selegilina}

As propargilaminas mostram-se úteis tanto na inibição da oxidação enzimática de DA como no processo não enzimático. Neste sentido, clorgilina, selegilina e pargilina (Figura 2) são capazes de inibir a oxidação não enzimática de DA in vitro e apresentam propriedades antioxidantes frente aos radicais hidroxilas. ${ }^{40}$ Dentre as propargilaminas, a selegilina tem sido um dos IMAO mais estudados, principalmente devido ao seu vasto espectro de atividades neuroprotetoras e neurorrestauradoras. ${ }^{41}$ Selegilina (L-Deprenyl ${ }^{\circledR}$; Eldery $\left.^{\circledR}\right)$ [(R)- $N$-metil- $N$-(1-fenilpropal-2-il)prop-1-in-3-amina] é um inibidor não competitivo e irreversível de MAO-B, que foi desenvolvido inicialmente como um antidepressivo e logo se tornou um sucesso no tratamento da DP. ${ }^{41} \mathrm{Na} \mathrm{DP}$, selegilina inibe irreversivelmente MAO-B através da reação entre a unidade flavina da enzima e a unidade acetilênica do fármaco. ${ }^{31}$ Devido as suas propriedades neuroprotetoras, selegilina tem sido também utilizada no tratamento da doença de Alzheimer, na qual apresenta efeito benéfico sobre o desempenho cognitivo. ${ }^{42}$

A atividade neuroprotetora da selegilina pode ser evidenciada por sua capacidade em bloquear o efeito do MPTP e proteger neuroblastomas de danos ao DNA induzidos por óxido nítrico e peróxinitrito, este último sugerindo que selegilina pode ser neuroprotetora aos neurônios dopaminérgicos através de sua atividade antiapoptótica. ${ }^{43,44}$ Como IMAO, selegilina previne o estresse oxidativo decorrente do processo de oxidação da DA, resultando na redução na produção de peróxido de hidrogênio durante essa reação. ${ }^{45}$

A selegilina possui uma ligação acetilênica capaz de reagir com radicais peroxila, seja pela abstração de hidrogênio ou por adição, o 
que resulta no término da reação radicalar em cadeia. ${ }^{46}$ Além disso, a unidade acetilênica permanece como principal região responsável pela atividade antioxidante da molécula. Estudos teóricos utilizando-se $\mathrm{N}, \mathrm{N}$-dimetilpropargilamina como modelo indicaram uma significante atividade radical scavenger da selegilina. ${ }^{47} \mathrm{~A}$ região da molécula reconhecida como aceptora de radicais foi identificada como sendo a unidade acetilênica, capaz de sequestrar tanto radicais ${ }^{\circ} \mathrm{OH}$ quanto radicais ${ }^{\circ} \mathrm{OOH}$, enquanto o anel aromático presente no fármaco parece contribuir para a sua biodisponibilidade e farmacocinética. ${ }^{47}$

Outro fator importante que tem sido negligenciado no estudo do efeito da selegilina sobre a oxidação não enzimática da DA é a grande reatividade apresentada entre quinonas, geradas na oxidação da DA, e aminas terciárias (selegilina), o que poderia, em parte, explicar o mecanismo antioxidante da selegilina. Aminas terciárias são oxidadas por quinonas à temperatura ambiente, sendo esta reação fortemente influenciada por fatores estruturais tanto da amina quanto da quinona.$^{48} \mathrm{Tem}$-se proposto que a susceptibilidade à oxidação de aminas por quinonas está relacionada com os potenciais redox das quinonas e com a propensão de quinonas em formar complexos de transferência de carga com a amina. Para a oxidação de aminas terciárias por quinonas, a formação do complexo de transferência de carga parece preceder a etapa de oxidação, sendo ambas as etapas dependentes de um alto potencial redox da quinona. ${ }^{49}$ Estes dados mostram que a interação de aminas terciárias com quinonas, formando complexos de transferência de carga, é promovida pela presença de anéis aromáticos ligados diretamente ao nitrogênio da amina e pela acessibilidade estérica ao átomo de nitrogênio da amina, sendo que a oxidação da amina ocorrerá caso os reagentes apresentem potenciais redox apropriados. ${ }^{48,49}$ No conjunto, estes dados podem ser utilizados no planejamento racional de novos compostos, derivados da selegilina, com ação antioxidante e com atividade inibitória sobre MAO-B.

\section{A DOENÇA DE PARKINSON E $\alpha$-SINUCLEÍNA}

A hipótese termodinâmica para o enovelamento proteico baseia-se no fato de que, durante o enovelamento, ocorre uma diminuição da energia livre até que seja alcançada uma estrutura correspondente ao mínimo de energia livre global, a despeito da existência de mínimos locais de energia (estados metaestáveis) - que correspondem a estruturas cineticamente estáveis (Figura 3). Entre os fatores que contribuem para o enovelamento proteico estão o efeito hidrofóbico $(\mathrm{EH}),{ }^{50}$ relacionado com o aumento de entropia que ocorre quando resíduos hidrofóbicos são removidos do contato com a água, e a formação de ligações de hidrogênios (LH) intramoleculares envolvendo tanto grupos polares da cadeia principal como das cadeias laterais, quando estes não se encontram expostos à água. ${ }^{51}$ Estes dois fatores, EH e LH, em maior ou menor extensão, contrabalançam a perda de entropia conformacional (EC) associada aos graus de liberdade da molécula que são drasticamente reduzidos durante o enovelamento. Assim, a estrutura nativa da proteína é, em geral, apenas marginalmente mais estável que as estruturas desenoveladas, o que pode ser verificado pelo baixo valor do $\Delta \mathrm{G}$ de desenovelamento $(\Delta \mathrm{Gu})$ apresentado pela maioria das proteínas (Figura 3, à esquerda).

Sob certas condições (mutações, aumento da concentração da proteína ou presença de ligantes específicos), uma via alternativa para o enovelamento proteico pode ser favorecida, onde predominam interações intermoleculares ao invés de intramoleculares, levando à associação proteína-proteína e agregação (Figura 3, à direita). ${ }^{52}$ $\mathrm{O}$ mau enovelamento proteico ocorre predominantemente quando estados cineticamente estáveis (metaestáveis), que correspondem a intermediários do enovelamento, são favorecidos. Estes estados apresentam um alto conteúdo de resíduos hidrofóbicos expostos à água e, neste caso, a interação proteína-proteína é acompanhada de

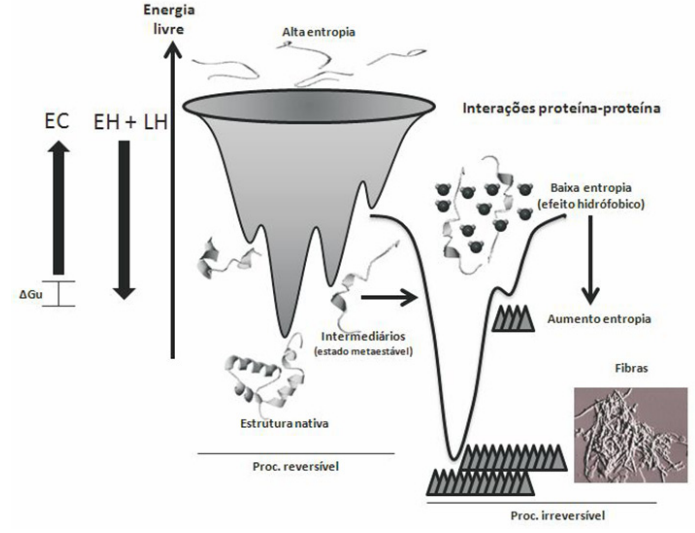

Figura 3. Hipótese termodinâmica para o enovelamento proteico. Entre os fatores que contribuem para o enovelamento de proteínas estão o efeito hidrofóbico (EH) e a formação de ligações de hidrogênio $(\mathrm{LH})$ intramoleculares, os quais contrabalançam a perda de entropia conformacional (EC). A estrutura nativa da proteína é apenas marginalmente mais estável que as estruturas desenoveladas $[\Delta G$ de desenovelamento $(\Delta G u)]$. À direita é mostrada uma via alternativa para o enovelamento proteico onde predominam interações intermoleculares, levando à associação proteína-proteína, que resulta na formação de agregados proteicos

um aumento de entropia (efeito hidrofóbico). O produto final deste processo é, muitas vezes, a formação de agregados insolúveis, sejam estes amorfos ou estruturados (fibras) - estes últimos caracterizados por uma alta estabilidade termodinâmica. Portanto, ao contrário do processo de enovelamento (reversível), a agregação de proteínas é um processo irreversível.

O mau enovelamento de proteínas, levando à formação de agregados proteicos potencialmente tóxicos para as células, parece ser um mecanismo comum em várias doenças neurodegenerativas. ${ }^{9}$ Alguns exemplos de doenças onde a formação de agregados proteicos se constitui num fator chave na origem e/ou progressão da doença são: a doença de Alzheimer (agregação do peptídeo b-amiloide e da proteína tau), encefalopatias espongiformes (agregação da proteína do príon), doença de Huntington (agregação da proteína huntingtina), ataxia espinocerebelar tipo-3 ou doença de Machado-Joseph (agregação da proteína ataxina-3).

No caso da DP, a perda dos neurônios dopaminérgicos é acompanhada pelo desenvolvimento de inclusões intracelulares, chamadas de Corpos de Lewy, compostas principalmente por agregados fibrilares da proteína $\alpha$-sinucleína. ${ }^{53}$ Nestas inclusões, a $\alpha$-sinucleína encontram-se ubiquitinada e fosforilada. O processo de ubiquitinação ocorre durante a via de degradação de proteínas (via proteossomal), principalmente aquelas incorretamente enoveladas. Além da DP, $\alpha$-sinucleína é o componente majoritário das lesões associadas a várias outras doenças neurológicas incluindo atrofia múltipla, a doença de Hallervorden-Spatz, variante da doença de Alzheimer com Corpos de Lewy e Demência com Corpos de Lewy. Estas doenças são caracterizadas por inclusões celulares de agregados de $\alpha$-sinucleína, formando Corpos de Lewy na DP, placas amilóidicas na doença de Alzheimer, inclusões glial-citoplasmáticas nos terminais pré-sinápticos em Atrofia Múltipla e em Esclerose Lateral Amiotrófica. $^{54}$

Fisiologicamente, demonstrou-se que a $\alpha$-sinucleína pode ser encontrada como um tetrâmero com estrutura em hélices ou na forma de um monômero desenovelado, o qual parece estar envolvido no processo de mau enovelamento e agregação. ${ }^{55,56} \mathrm{Na} \mathrm{DP}$, o envolvimento da $\alpha$-sinucleína é evidenciado, principalmente, devido ao fato de duas mutações, Ala30Pro e Ala53Thr, estarem ligadas a casos da doença em grupos de indivíduos mais jovens. ${ }^{8}$ 
Embora $\alpha$-sinucleína com estrutura fibrilar seja o maior componente dos Corpos de Lewy, ${ }^{57}$ alguns dados sugerem que estas estruturas podem ser inertes ou até mesmo protetoras na DP. Primeiro, no modelo dos camundongos transgênicos superexpressando $\alpha$-sinucleína, observa-se que os sintomas característicos de DP são acompanhados pela formação de inclusões citoplasmáticas não fibrilares de $\alpha$-sinucleína. ${ }^{58}$ Segundo, demonstrou-se que estruturas intermediárias (protofibras) da agregação da $\alpha$-sinucleína são capazes de promover permeabilização de vesículas e esta propriedade é sensível às mutações da proteína ligadas à $\mathrm{DP}{ }^{59}$ Além disso, mutações na $\alpha$-sinucleína associadas à DP aumentam a formação de protofibras in vitro quando comparadas à proteína selvagem. ${ }^{57}$

Evidências recentes sugerem que a $\alpha$-sinucleína atue nos terminais pré-sinápticos como uma cochaperona na formação do complexo envolvendo a proteína receptora do fator de acoplamento sensível à etilmaleimida (SNARE - soluble N-ethylmaleimide-sensitive factor attachment protein receptor), a qual desempenha um papel fundamental na mediação do tráfego de vesículas nas células, principalmente na catálise do processo de fusão vesicular. ${ }^{60}$ Neste processo, a $\alpha$-sinucleína se ligaria diretamente à proteína SNARE sinaptobrevina-2/VAMP2 (vesicle-associated membrane protein 2), promovendo a formação do complexo. Portanto, a perda da $\alpha$-sinucleína funcional, que ocorreria devido ao processo de agregação, poderia representar um evento crítico na neuropatogênese da DP sob o ponto de visto do mau enovelamento da proteína. Neste contexto, a prevenção do mau enovelamento/agregação da $\alpha$-sinucleína seria o objetivo principal da intervenção farmacológica.

Por outro lado, tem sido demonstrado que $\alpha$-sinucleína na forma fibrilar ou protofibrilar é capaz de recrutar a $\alpha$-sinucleína solúvel e causar prejuízos à função sináptica dos neurônios, com consequente neurodegeneração. ${ }^{61}$ Assim, moléculas capazes de se ligarem à $\alpha$-sinucleína, estabilizando a espécie funcional (monômero desenovelado ou tetrâmero), prevenindo o processo de mau enovelamento e formação de fibras, poderiam representar potenciais fármacos antiparkinsonianos.

Demonstrou-se que produtos da oxidação de catecolaminas, como o dopaminocromo (Figura 1), são capazes de inibir a fibrilação da $\alpha$-sinucleína, bem como promover a desagregação das fibras pré-existentes, levando à formação de estruturas agregadas (protofibras) - potencialmente mais tóxicas que as fibras. ${ }^{62} \mathrm{Se}$ o processo de fibrilação resulta na formação de espécies com menor toxicidade, quando comparadas com as protofibras, compostos capazes de estabilizar esses intermediários pré-fibrilares podem representar fatores chaves na etiologia da DP. Assim, podem-se definir compostos com atividade antiamiloidogênica como aqueles que inibem a agregação da $\alpha$-sinucleína, impedindo a formação de espécies tóxicas, sejam elas protofibras ou fibras.

Com o objetivo de identificar moléculas capazes de inibir a formação de fibras amiloides de $\alpha$-sinucleína, uma biblioteca de compostos comercialmente disponíveis foi triada pelo grupo do Dr. P. T. Lansbury, Jr. ${ }^{62}$ A grande maioria destes compostos eram catecóis e hidroquinonas, incluindo DA, $L$-dopa, noradrenalina e adrenalina. Nestes estudos, a atividade inibitória da fibrilização de $\alpha$-sinucleína por DA e $L$-dopa foi revertida pela adição de antioxidantes (metabissulfito de sódio), sugerindo que a forma oxidada destas moléculas era a responsável pelo efeito inibitório. Além de catecóis, muitos flavonoides inibem a formação de fibras de $\alpha$-sinucleína. ${ }^{63} \mathrm{DA}$ e polifenóis são facilmente oxidados às suas formas quinonas quando expostos ao oxigênio atmosférico. ${ }^{64}$

Follmer e colaboradores mostraram que DA interage com $\alpha$-sinucleína e modula diferentemente a estabilidade de agregados gerados a partir das formas selvagem ou mutantes da proteína ${ }^{65} \mathrm{Na}$ ausência de DA, protofibras de $\alpha$-sinucleína exibem estabilidades semelhantes quando submetidas à dissociação por pressão hidrostática. Entretanto, protofibras geradas a partir das variantes da $\alpha$-sinucleína (Ala30Pro ou Ala53Thr), quando em presença de DA, apresentaram uma maior estabilidade quando comparadas às protofibras geradas a partir da forma selvagem da proteína. Assim, DA apresentaria um efeito estabilizador sobre as espécies intermediárias da agregação da $\alpha$-sinucleína, supostamente mais tóxicas, apenas quando estas são formadas pelas mutantes da proteína. Estes dados sugerem uma ligação entre as mutações na $\alpha$-sinucleína, relacionadas com DP em indivíduos mais jovens, DA e a formação de protofibras.

Muitos agentes antiparkinsonianos inibem ou retardam a formação de fibras tanto da $\alpha$-sinucleína como do peptídeo $\beta$-amiloide, este último envolvido na doença de Alzheimer. ${ }^{66,67}$ Além disso, estes compostos são capazes de desestabilizar fibras pré-existentes. Assim, demonstrou-se que estas moléculas apresentam a seguinte ordem de atividade antiamiloidogênica: DA $>$ selegilina $>L$-dopa $>$ pergolida $>$ bromocriptina. Embora o mecanismo da atividade antiamiloidogênica seja desconhecido, acredita-se que estes compostos e outros estruturalmente relacionados sejam moléculas-chave no desenvolvimento de terapias tanto para DP como para outras doenças amiloidogênicas. No caso do peptídeo $\beta$-amiloide, especula-se que a atividade antiamiloidogênica destes compostos esteja relacionada à unidade 3,4-di-hidroxifenil, que se ligaria de forma específica ao peptídeo e, subsequentemente, inibiria a sua polimerização ou, no caso de fibras já formadas, desestabilizaria a conformação em folhas $\beta$ presentes nas fibras maduras. ${ }^{66}$

Visto que selegilina previne (ou retarda) o processo de oxidação da DA, uma importante questão é de que forma este fármaco influenciaria no sistema $\alpha$-sinucleína-DA, quando estes dois fármacos são combinados na terapia da DP. Diante dos dados mostrando o efeito da DA sobre a agregação da $\alpha$-sinucleína previamente discutidos, ${ }^{65}$ uma abordagem de tratamento racional para DP deverá considerar a ação combinada destes fármacos na doença.

O processo de fibrilação da $\alpha$-sinucleína ocorre segundo uma cinética sigmoidal: uma fase lenta (fase "lag"), onde ocorre a formação do núcleo que dará origem à fibra, seguida de uma fase exponencial (fase "log"), relacionada com o crescimento da fibra. Demonstrou-se que selegilina interage com a $\alpha$-sinucleína e retarda a formação de fibras, aumentando o período da "fase lag" da cinética de agregação, gerando principalmente agregados amorfos que se mostraram inertes em culturas de neurônios dopaminérgicos. ${ }^{68}$ Selegilina estaria assim interferindo com a formação do núcleo envolvido na fase lenta da cinética de fibrilação. Quando combinada com DA, que promove a formação de protofibras tóxicas, ${ }^{65}$ selegilina previne a formação dos agregados tóxicos, o que pode representar um importante efeito quando o IMAO e $L$-dopa (que será convertida em DA) são coadministradas. No conjunto, estes dados sugerem que selegilina possa ser um fármaco multifuncional sob o ponto de vista da inibição da MAO e da inibição da formação de agregados tóxicos de $\alpha$-sinucleína.

Outros IMAO parecem apresentar efeito modulador sobre a fibrilação da $\alpha$-sinucleína, como é o caso de algumas 1,4-naftoquinonas. Menadiona (vitamina K3) e 1,4-naftoquinona, inibidores reversíveis de MAO-A e MAO-B, modulam a cinética de agregação da $\alpha$-sinucleína, inibindo a formação de fibras, o que resulta em agregados amorfos e pequenas fibras. ${ }^{69}$ Resultados semelhantes foram observados para as vitaminas K filoquinona (vitamina K1) e menaquinona (vitamina K2), embora estas moléculas não apresentem atividade sobre MAO. Menadiona e 1,4-naftoquinona também apresentam efeito desagregante, gerando principalmente fibras fragmentadas. No conjunto, estes dados sugerem que 1,4-naftoquinona parece se constituir numa importante molécula líder para o desenvolvimento de fármacos que visem interferir tanto na formação/desagregação de fibras de $\alpha$-sinucleína como na inibição reversível de MAO. 


\section{CONSIDERAÇÕES FINAIS}

O desenvolvimento de fármacos com atividade neuroprotetora, visando novas estratégias terapêuticas para doenças neurodegenerativas, permanece como um dos grandes desafios da indústria farmacêutica. No caso da DP, o desenvolvimento de fármacos multialvos ou multifuncionais deve considerar, principalmente, os aspectos químicos relacionados às atividades antioxidantes (proteção frente ao estresse oxidativo associado ao metabolismo de DA), inibitórias sobre MAO (visando um aumento nos níveis de certos neurotransmissores, principalmente DA e diminuição da produção de peróxido de hidrogênio) e antiamiloidogênicas (inibição da formação de agregados proteicos tóxicos) das moléculas em estudo. O entendimento dos mecanismos envolvidos nestas diferentes atividades é crucial para o planejamento racional de novos fármacos e, neste contexto, os grandes desafios residem, principalmente, na elucidação das relações entre os intrincados eventos que ocorrem durante o processo de neurodegeneração.

\section{MATERIAL SUPLEMENTAR}

As Figuras 1S, 2S e 3S estão disponíveis em http://quimicanova. sbq.org.br, em arquivo pdf, com acesso livre.

\section{AGRADECIMENTOS}

À Fundação de Amparo à Pesquisa do Estado do Rio de Janeiro (FAPERJ); Souza-Cruz S.A.; CNPq- Instituto Nacional de C,T\&I em Materiais Complexos Funcionais; International Foundation for Science (IFS).

\section{REFERÊNCIAS}

1. Lees, A. J.; Hardy, J.; Revesz, T.; Lancet 2009, 373, 2055.

2. Poewe, W.; Antonini, A.; Zijlmans, J. C.; Burkhard, P. R.; Vingerhoets, F.; Clin. Interv. Aging 2010, 5, 229.

3. Sozio, P.; Cerasa, L. S.; Abbadessa, A.; Di Stefano, A.; Expert Opin. Drug Discov. 2012, 7, 385.

4. Lippman, S. B.; Nash, K.; Drug Safety 1990, 5, 195.

5. Da Prada, M.; Kettler, R.; Keller, H. H.; Burkard, W. P.; Haefely, W. E.; J. Neural Transm. Suppl. 1989, 28, 5.

6. Volles, M. J.; Lansbury, P. T. Jr.; Biochemistry 2003, 42, 7871.

7. Polymeropoulos, M. H.; Lavedan, C.; Leroy, E.; Ide, S. E.; Dehejia, A.; Dutra, A.; Pike, B.; Root, H.; Rubenstein, J.; Boyer, R.; Stenroos, E. S.; Chandrasekharappa, S.; Athanassiadou, A.; Papapetropoulos, T.; Johnson, W. G., Lazzarini, A. M.; Duvoisin, R. C.; Di Iorio, G.; Golbe, L. I.; Nussbaum, R. L.; Science 1997, 276, 2045.

8. Krüger, R.; Kuhn, W.; Müller, T.; Woitalla, D.; Graeber, M.; Kösel, S.; Przuntek, H.; Epplen, J. T.; Schöls, L.; Riess, O.; Nat. Genet. 1998, 18, 106.

9. Chiti, F.; Dobson, C. M.; Annu. Rev. Biochem. 2006, 75, 333.

10. Jenner, P.; Ann. Neurol. 2003, 53, 26.

11. Zorov, D. B.; Bannikova, S. Y.; Belousov, V. V.; Vyssokikh, M. Y.; Zorova, L. D.; Isaev, N. K.; Krasnikov, B. F.; Plotnikov, E. Y.; Biochemistry (Moscow) 2005, 70, 215.

12. Ray, P. D.; Huang, B. W.; Tsuji, Y.; Cell Signal. 2012, 24, 981.

13. Fornstedt, B.; Brun, A.; Rosengren, E.; Carlsson, A.; J. Neural Transm. Park. Dis. Dement. Sect. 1989, 1, 279.

14. Sulzer, D.; Zecca, L.; Neurotox. Res. 2000, 1, 181.

15. Klegeris, A.; Korkina, L. G.; Greenfield, S. A.; Free Radical Biol. Med. 1995, 18, 215.

16. Anderson, D. G.; Mariappan, S. V.; Buettner, G. R.; Doorn, J. A.; J. Biol. Chem. 2011, 286, 26978.
17. Binda, C.; Newton-Vinson, P.; Hubálek, F.; Edmondson, D. E.; Mattevi, A.; Nat. Struct. Biol. 2002, 9, 22.

18. Javitch, J. A.; D’Amato, R. J.; Strittmatter, S. M.; Snyder, S. H.; Proc. Natl. Acad. Sci. U. S. A. 1985, 82, 2173.

19. Edmondson, D. E.; Binda, C.; Wang, J.; Upadhyay, A. K.; Mattevi, A.; Biochemistry 2009, 48, 4220.

20. Bach, A. W.; Lan, N. C.; Johnson, D. L.; Abell, C. W.; Bembenek, M. E.; Kwan, S. W.; Seeburg, P. H.; Shih, J. C.; Proc. Natl. Acad. Sci. U. S. A. 1988, 85, 4934.

21. Youdim, M. B.; Bakhle, Y. S.; Br. J. Pharmacol. 2006, 147, 287.

22. Guay, D. R.; Am. J. Geriatr. Pharmacother. 2006, 4, 330.

23. Riederer, P.; Lachenmayer, L.; Laux, G.; Curr. Med. Chem. 2004, 11, 2033.

24. Youdim, M. B.; Weinstock, M.; Neurotoxicology 2004, 25, 243.

25. Tatton, W. G.; Chalmers-Redman, R. M.; Neurology 1996, 47, 171.

26. Youdim, M. B.; Edmondson, D.; Tipton, K. F.; Nat. Rev. Neurosci. 2006 7, 295.

27. Riederer, P.; Youdim, M. B.; J. Neurochem. 1986, 46, 1359.

28. Son, S. Y.; Ma, J.; Kondou, Y.; Yoshimura, M.; Yamashita, E.; Tsukihara, T.; Proc. Natl. Acad. Sci. U. S. A. 2008, 105, 5739.

29. Binda, C.; Wang, J.; Pisani, L.; Caccia, C.; Carotti, A.; Salvati, P.; Edmondson, D. E.; Mattevi, A.; J. Med. Chem. 2007, 50, 5848.

30. Binda, C.; Mattevi, A.; Edmondson, D. E.; J. Biol. Chem. 2002, 277, 23973.

31. De Colibus, L.; Li M.; Binda, C.; Lustig, A.; Edmondson, D. E.; Mattevi, A.; Proc. Natl. Acad. Sci. U. S. A. 2005, 102, 12684.

32. Altomare, C.; Cellamare, S.; Summo, L.; Catto, M.; Carotti, A.; Thull, U.; Carrupt, P. A.; Testa, B.; Stoeckli-Evans, H.; J. Med. Chem. 1998, $41,3812$.

33. Castagnoli, K. P.; Steyn, S. J.; Petzer, J. P.; van der Schyf, C. J.; Castagnoli, N. J.; Chem. Res. Toxicol. 2001, 14, 523.

34. Coelho-Cerqueira, E.; Netz, P. A.; Diniz, C.; Petry do Canto, V.; Follmer, C.; Bioorg. Med. Chem. 2011, 19, 7416.

35. Nandigama, R. K.; Miller, J. R.; Edmondson, D. E.; Biochemistry 2001, $40,14839$.

36. Nagatsu, T.; Sawada, M.; J. Neural. Transm. Suppl. 2006, 71, 53.

37. Toprakçí, M.; Yelekçi, K.; Bioorg. Med. Chem. Lett. 2005, 15, 4438.

38. Thomson, R. H.; Naturally Occurring Quinones, Springer: London, 1996.

39. Youdim, M. B. H.; Gross, A.; Finberg, J. P. M.; Br. J. Pharmacol. 2001, 132,500 .

40. Chiueh, C. C.; Huang, S. J.; Murphy, D. L.; J. Neural Transm. Suppl. 1994, 41, 189.

41. Lange, K. W.; Riederer, P.; Youdim, M. B.; Clin. Pharmacol. Ther. 1994, $56,734$.

42. Tolbert, S. R.; Fuller, M. A.; Ann. Pharmacother. 1996, 30, 1122.

43. Cohen, G.; Pasik, P.; Cohen, B.; Leist, A.; Mytilineou, C.; Yahr, M. D.; Eur. J. Pharmacol. 1984, 106, 209.

44. Maruyama, W.; Takahashi, T.; Naoi, M.; J. Neurochem. 1998, 70, 2510.

45. De Marchi, U.; Pietrangeli, P.; Marcocci, L.; Mondovì, B.; Toninello, A.; Biochem. Pharmacol. 2003, 66, 1749.

46. Thomas, C. E.; Huber, E. W.; Ohlweiler, D. F.; Free Radical Biol. Med. 1997, 22, 733 .

47. Nakai, S.; Yoneda, F.; Theor. Chem. Acc. 2000, 104, 398.

48. Buckley, D.; Dunstan, S.; Henbest, H. B.; J. Chem. Soc. 1957, 4880.

49. Eckert, T. S.; Bruice, T. C.; J. Am. Chem. Soc. 1983, 105, 4431.

50. Meyer, E. E.; Rosenberg, K. J.; Israelachvili, J.; Proc. Natl. Acad. Sci. U. S. A. 2006, 103, 15739.

51. Bolen, D. W.; Rose, G. D.; Ann. Rev. Biochem. 2008, 77, 339.

52. Mousseau, N.; Derreumaux, P.; Front. Biosci. 2008, 13, 4495.

53. Breydo, L.; Wu, J. W.; Uversky, V. N.; Biochim. Biophys. Acta 2012, $1822,261$.

54. Lee, S. J.; Lim, H. S.; Masliah, E.; Lee, H. J.; Neurosci. Res. 2011, 70, 339. 
55. Bartels, T.; Choi, J. G.; Selkoe, D. J.; Nature 2011, 477, 107.

56. Fauvet, B.; Mbefo, M. K.; Fares, M. B.; Desobry, C.; Michael, S.; Ardah, M. T.; Tsika, E.; Coune, P.; Prudent, M.; Lion, N.; Eliezer, D.; Moore, D. J.; Schneider, B.; Aebischer, P.; El-Agnaf, O. M.; Masliah, E.; Lashuel, H. A.; J. Biol. Chem. 2012, 287, 15345.

57. Conway, K. A.; Harper, J. D.; Lansbury, P. T. Jr.; Biochemistry 2000, 39, 2552.

58. Masliah, E.; Rockenstein, E.; Veinbergs, I.; Mallory, M.; Hashimoto, M.; Takeda, A.; Sagara, Y.; Sisk, A.; Mucke, L.; Science 2000, 287, 1265.

59. Volles, M. J.; Lansbury, P. T. Jr.; Biochemistry 2002, 41, 4595.

60. Burré, J.; Sharma, M.; Tsetsenis, T.; Buchman, V.; Etherton, M. R.; Südhof, T. C.; Science 2010, 329, 1663.

61. Volpicelli-Daley, L. A.; Luk, K. C.; Patel, T. P.; Tanik, S. A.; Riddle, D. M.; Stieber, A.; Meaney, D. F.; Trojanowski, J. Q.; Lee, V. M.; Neuron 2011, 72, 57.

62. Conway, K. A.; Rochet, J. C.; Bieganski, R. M.; Lansbury, P. T. Jr.; Science 2001, 294, 1346.
63. Caruana, M.; Högen, T.; Levin, J.; Hillmer, A.; Giese, A.; Vassallo, N.; FEBS Lett. 2011, 585, 1113.

64. Li, H. T.; Lin, D. H.; Luo, X. Y.; Zhang, F.; Ji, L. N.; Du, H. N.; Song, G. Q.; Hu, J.; Zhou, J. W.; Hu, H. Y.; FEBS J. 2005, 272, 3661.

65. Follmer, C.; Romão, L.; Einsiedler, C. M.; Porto, T. C. R.; Lara, F.; Monçores, M.; Weissmüller, G.; Lashuel, H.; Lansbury, P. T. Jr.; MouraNeto, V.; Silva, J. L.; Foguel, D.; Biochemistry 2007, 46, 472.

66. Ono, K.; Hasegawa, K.; Naiki, H.;Yamada, M.; Neurochem. Int. 2006, 48,275 .

67. Ono, K.; Hirohata, M.; Yamada, M.; J. Neurosci. Res. 2007, 85, 1547.

68. Braga, C. A.; Follmer, C.; Palhano, F. L.; Khattar E.; Freitas, M. S.; Romão L.; Di Giovanni, S.; Lashuel, H. A.; Silva, J. L.; Foguel, D.; J. Mol. Biol. 2011, 405, 254.

69. da Silva, F.L.; Coelho-Cerqueira, E.; Freitas, M. S.; Gonçalves, D. L.; Costa, L. T.; Follmer, C.; Neurochem. Int. 2013, 62, 103 
FÁRMACOS MULTIFUNCIONAIS: MONOAMINA OXIDASE E $\alpha$-SINUCLEÍNA COMO ALVOS TERAPÊUTICOS NA DOENÇA DE PARKINSON

\section{Cristian Follmer*}

Departamento de Físico-Química, Instituto de Química, Centro de Ciências Matemáticas e da Natureza, Universidade Federal do Rio de Janeiro, 21941-909 Rio de Janeiro - RJ, Brasil

Heleno J. C. Bezerra Netto

Instituto Nacional de Propriedade Industrial, Praça Mauá, 7, 20081-240 Rio de Janeiro - RJ, Brasil

A

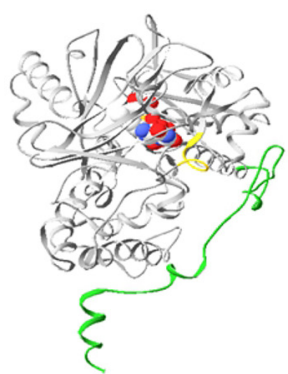

C

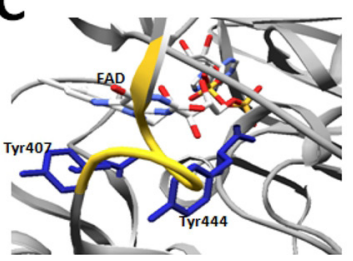

B

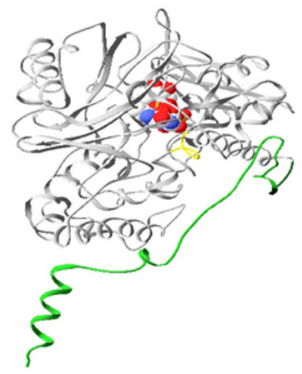

D

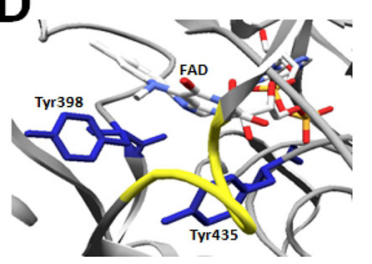

Figura 1S. Estruturas das enzimas MAO-A e MAO-B. A subunidade monomérica (cadeia A) da MAO-A (PDB: 2Z5Y) e MAO-B (PDB: 2BYB) são mostradas em $A$ e B, respectivamente. O grupo FAD [representado no modo CPK (Corey-Pauling-Koltun)], a região envolvida no ancoramento à membrana mitocondrial externa (em verde) e a alça 210-216 (em MAO-A) e 201-206 (em MAO-B), envolvida na seletividade da enzima, foram destacadas. Em C e D são mostrados o cofator FAD e as duas tirosinas (em azul) que constituem a "gaiola aromática", Tyr407/Tyr444 em MAO-A e Tyr398/Tyr435 em MAO-B, respectivamente. As imagens foram construídas utilizando-se o programa SwissPDB Viewer

A
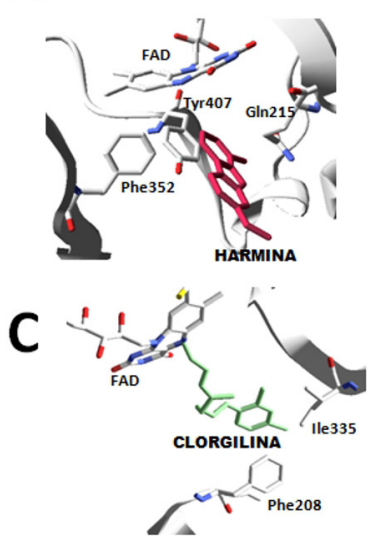

B
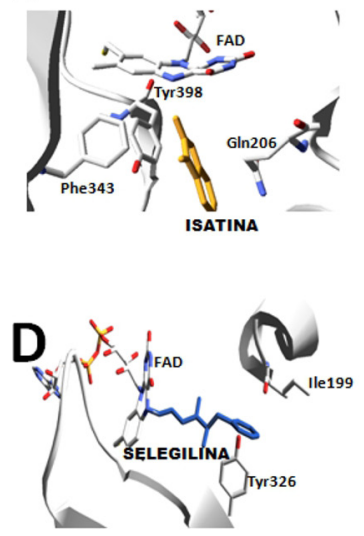

Figura $2 S$. Estrutura da MAO-A e MAO-B em presença de inibidores reversíveis e irreversíveis. (A) Interação dos inibidores reversíveis harmina (em magenta) no sítio catalítico da MAO-A (PDB: 2Z5X); (B) Isatina (em amarelo) na MAO-B (PDB: 1OJA); (C) MAO-A ligada ao inibidor irreversível clorgilina (em verde) (PDB: 2BXR); (D) MAO-B ligada ao inibidor irreversível selegilina (em azul) (PDB: 2BYB). As imagens foram construídas utilizando-se o programa SwissPDB Viewer 

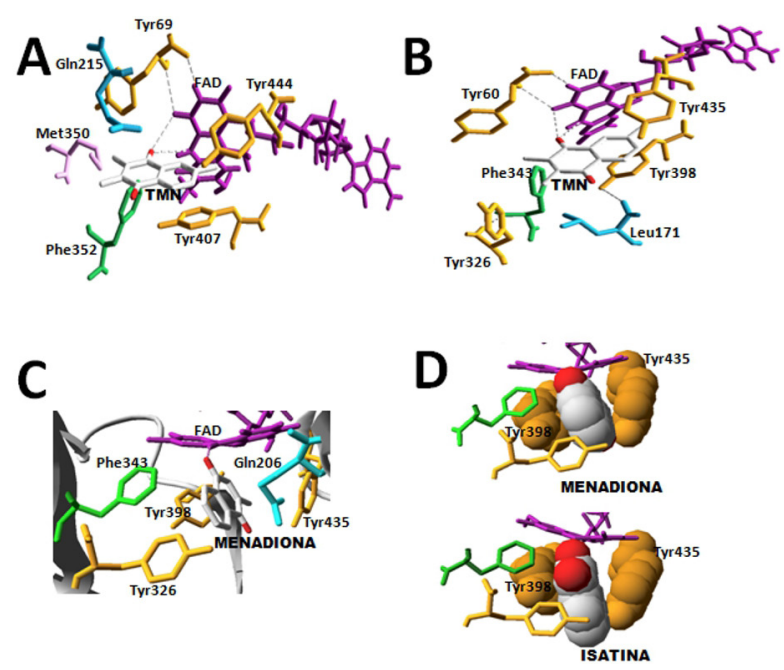

Figura 3S. Inibição da MAO pelas 1,4-naftoquinonas: TMN e menadiona. O inibidor reversível e não seletivo TMN interage com a porção flavina do FAD da MAO-A (A) e MAO-B (B). Em (C), menadiona interage com resíduos do sítio catalítico da MAO-B, com destaque para a formação de uma ligação de hidrogênio com o grupamento flavina do FAD. As cadeias laterais dos resíduos Tyr398 e Tyr435 da MAO-B estão envolvidas na estabilização do anel 1,4-naftoquinona da menadiona no sítio catalítico, similarmente ao observado com o inibidor reversível isatina $(D)$. As imagens foram construídas utilizando-se o programa SwissPDB Viewer 